\title{
Optimization of an In Vitro Dissolution Test Method for Inhalation Formulations
}

\author{
Yoen-Ju Son ${ }^{1}$, Michelle Horng ${ }^{1}$, Mark Copley², and Jason T. McConville ${ }^{1, *} \quad$ e-mail:jtmcconville@mail.utexas.edu \\ 'College of Pharmacy, The University of Texas at Austin, Austin, TX, USA \\ ${ }^{2}$ Copley Scientific Limited, Nottingham, UK
}

\begin{abstract}
The aim of this research project was to investigate a potential standardized test method to characterize the dissolution properties of numerous formulation types available for pulmonary delivery. A commercially available dissolution tester was adapted for use as a testing apparatus by the incorporation of a membrane-containing holder. The holder was designed to enclose previously air-classified formulations so that they could be uniformly tested in the dissolution apparatus. Dissolution procedures, the apparatus, dose collection, medium, and test conditions were developed relying on USP General Chapter <1092>. To collect an active pharmaceutical ingredient (API) fraction from the devices for subsequent dissolution studies, aerodynamic particle separation on the membrane holder was achieved using the Next Generation Impactor (NGI) for two commercially available products, Ventolin HFA and Pulmicort Flexhaler. The dissolution profiles of budesonide (BD) and albuterol sulfate (AS) were successfully estimated by analyzing the amount of drug released from the membrane holder. This dissolution method may be applied to quality control studies for various inhalation products. In particular, the in vitro dissolution profiles of the drugs may provide an estimate of their dispersion characteristics, which directly relate to the device or aerosol performances.
\end{abstract}

\section{INTRODUCTION}

D issolution testing allows one to examine the drug release behavior of pharmaceutical dosage forms in vitro to differentiate formulation types and perhaps give an estimate of dissolution behavior in vivo. Dissolution testing is routinely used in quality control (QC) studies such as batch-to-batch consistency, stability, and detection of manufacturing deviations. While there are many standardized dissolution test methods for solid dosage forms such as tablets and capsules, there is no universally accepted method for estimating the dissolution behavior of inhaled active ingredients, although many dissolution methods for testing aerosols have been investigated (1). Designing a standardized method applicable to the lung is not an easy task, because the lung has several unique features that are difficult to replicate in vitro, such as the extremely small amount of aqueous fluid and the presence of endogenous lung surfactants $(1,2)$.

For inhalation products, the most important step for in vitro performance testing is the delivery of a given API from a specified delivery device and its deposition using a pharmaceutical impactor/impinger to estimate the actual dose delivered to the target site of the lung. In pulmonary drug delivery, it is well-accepted that particles within the size range of 1-5 $\mu \mathrm{m}$ can be successfully delivered to the targeted deep lung. Only a fraction of the API emitted from standard delivery devices is usually delivered to this target site, due to the fine particle size distribution for most inhaler products (2). Thus, an ideal dissolution test

*Corresponding author procedure for inhalation formulations would involve particle classification followed by an evaluation of the dissolution behavior of those sorted drug particles that may deposit at various sites in the respiratory tract.

Experimental difficulties in dose collection exist due to very fine and electrostatic powder characteristics (1). Therefore, most existing dissolution procedures on powders have been performed with no aerodynamic classification, whereby formulations have been directly dispersed into an Apparatus 2 dissolution tester (3) or placed directly into a modified basket to prevent drug particles from escaping directly into the dissolution medium $(4,5)$. Formulations intended for pulmonary delivery are hard to disperse homogeneously into the vessel or basket, and dispersed particles stick on the vessel wall or paddle/basket during such dissolution tests. In addition, floating powders may be inadvertently collected during the sampling procedure. In an attempt to compensate for some of the shortfalls of this type of testing using commercial dissolution systems, several custom-built dissolution apparatus have been investigated. Davies and Feddah (6) modified a flow-through cell by direct incorporation of an HPLC pump. In another study that used a horizontal diffusion cell, powders were dispersed onto a hydrated membrane, and the dissolution rate was estimated by observation of the diffusion rate (7). In addition to these methods, the twin-stage impinger (TSI) (8), dissolution cell $(9,10)$, and shaking incubator $(11,12)$ apparatus were also modified for conducting in vitro dissolution studies for dry powders. Although these approaches do, in some way, make up for the drawbacks indicated above for a commercial dissolution apparatus, 
problems such as dose collection, adequate particle dispersion, and uniformity of particle size distribution are still evident. Therefore, no single in vitro test system has previously been described to be universally suitable for performing dissolution measurements of inhalation formulations.

This article describes the features of a newly developed membrane holder that was designed specifically to be incorporated into the Next Generation Impactor (NGI) for better dose collection performance than with a previously reported prototype membrane holder. In the previous study (13), a prototype membrane holder was used to assess the dissolution profiles of aerodynamically separated drug particles. Dose collection was achieved by aerodynamic separation into the NGI modified with a polycarbonate membrane. The dissolution profiles of a model drug were successively estimated by the amount of drug released from the membrane holder. It was clearly shown that there was a significant difference between the bulk formulation and an aerodynamically classified formulation in the dissolution profile. However, in the previous dissolution setup, modification of the NGI was required to collect dispersed particles on the membrane, and the whole dose collected on the membrane could not be used for dissolution testing due to a substantial limitation imparted by the prototype holder frame size. The aim of this study was to investigate a standardized dissolution procedure using the newly manufactured membrane holder and to characterize the dissolution properties of two different types of commercially available inhalation product.

\section{MATERIALS AND METHODS \\ Materials}

Ventolin HFA was purchased from GlaxoSmithKline (Research Triangle Park, NC) and a Pulmicort Flexhaler was purchased from AstraZeneca LP (Wilmington, DE). Budesonide (99\%) was purchased from Sigma Chemical Co. (St. Louis, MO). Albuterol sulfate, USP was purchased from Spectrum Chemical Co. (Gardena, CA). Dipalmitoyl phosphatidylcholine (DPPC) was purchased from Avanti Polar Lipid, Inc. (Alabaster, AL). Polycarbonate (PC) membranes of $0.05 \mu \mathrm{m}(76 \mathrm{~mm})$ were purchased from Whatman (Florham Park, NJ). Methanol (HPLC grade) and all other reagents were purchased from Sigma Chemical Co. (St. Louis, MO).

\section{Dissolution Apparatus}

A USP Apparatus 2, Hanson SR8-Plus dissolution test station (Hanson Co., Chatsworth, CA), was employed to conduct the dissolution study. A schematic diagram of modifications to the dissolution apparatus is shown in Figure 1.The two main components of the dissolution setup include the dissolution test station (Figure 1A) and a newly designed membrane holder (Copley Scientific Limited, Nottingham, UK) (Figure 1B). The membrane holder assembly was customized; it consists of an NGI
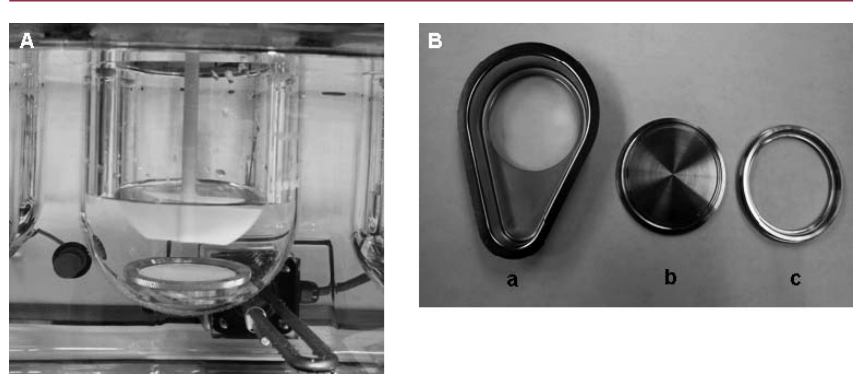

Figure 1. Schematic diagram of the dissolution apparatus. (A) Dissolution station and (B) membrane holder assembly: (a) NGI dissolution cup, (b) impaction insert, and (c) securing ring.

dissolution cup (a), a removable impaction inset (b), a securing ring (c), two sealing o-rings, and a PC membrane to function as a highly porous diffusional powderretaining layer.

\section{Dose Collection}

To select suitable particle size cutoff ranges for the subsequent dissolution study, aerodynamic particle separation was achieved using the NGI. Either the Ventolin HFA device or the Pulmicort Flexhaler device was actuated five times to obtain a quantifiable amount of drug. The $\mathrm{NGI}$ was operated at a flow rate of $30 \mathrm{~L} / \mathrm{min}$ for the Ventolin HFA and $60 \mathrm{~L} / \mathrm{min}$ for the Pulmicort Flexhaler. Air-classified particles from each dose-collection plate of the NGI that were not used in the dissolution studies were reconstituted with mobile phase and analyzed using a validated HPLC method $(14,15)$.

For the dissolution studies, the dissolution cup assembled with the impaction insert was placed in the $\mathrm{NGl}$, as shown in Figure 2A. Either the Ventolin HFA or the Pulmicort Flexhaler device was fired into the NGI at the same flow rate as above. Following actuation, the impaction insert was removed from the NGI dissolution cup for the dissolution test.

\section{Dissolution Media}

Simulated lung fluid (SLF), $0.2 \mathrm{M}$ phosphate buffer (pH 7.4), phosphate-buffered saline (PBS), modified PBS (mPBS) containing dipalmitoylphosphatidylcholine
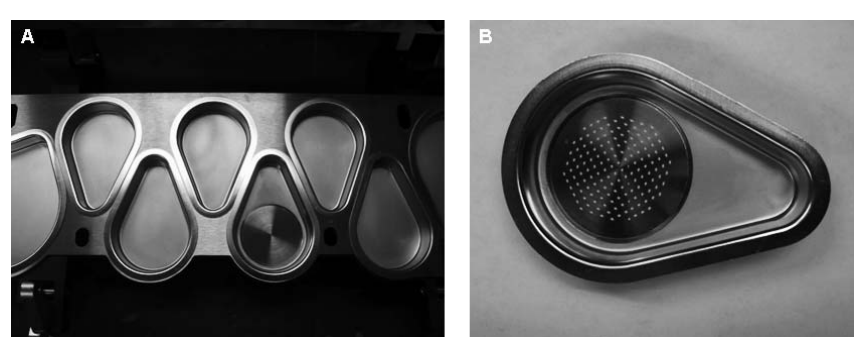

Figure 2. Modified next-generation impactor (NGI). (A) NGI setup before impingement. (B) Resulting impingement. 
(DPPC), and PBS containing polysorbate 80 (tPBS) were used in the dissolution studies.

To prepare the mPBS, DPPC ( $500 \mathrm{mg}$ ) was weighed into a $500-\mathrm{mL}$ round-bottom flask and dissolved in $100 \mathrm{~mL}$ of a chloroform/methanol (1:1) mixture. The solvent was evaporated by rotary evaporation (Buchi Corporation, New Castle, DE). The dry, thin film was rehydrated with $250 \mathrm{~mL}$ PBS at $55^{\circ} \mathrm{C}$ and rotated for $2 \mathrm{~h}$. The warm suspension was placed into a Branson 5500 sonic bath (Branson Ultrasonics, Danbury, CT) and sonicated at $55^{\circ} \mathrm{C}$ for $1 \mathrm{hr}$. The concentrated DPPC solution $(0.2 \%)$ was stored at $4{ }^{\circ} \mathrm{C}$. The prepared stock DPPC solution $(0.2 \%)$ was diluted 10 -fold with PBS before use.

\section{Dissolution Study}

To aerodynamically classify budesonide (BD) and albuterol sulfate (AS) from the Pulmicort Flexhaler device or the Ventolin HFA device, each formulation was fired into the NGI as previously described. The NGI dose plates were assembled as above (with dissolution cup). Following actuation for a given formulation, the impaction insert was removed from the NGI dissolution cup. A pre-soaked membrane was placed on the top and sealed in place with the securing ring of the new membrane holder. The sealed membrane holder was then placed into each dissolution vessel containing $300 \mathrm{~mL}$ of dissolution medium (Figure 1A). Dissolution testing was conducted at 50,75 , and $100 \mathrm{rpm}$. Aliquots ( $3 \mathrm{~mL}$ ) of dissolution media were withdrawn manually using a glass syringe at timed intervals of $5,15,30,60,90$, and 120 min for the BD samples and 5, 15,30,45, and 60 min for the AS samples. Collected dissolution samples of BD $(2 \mathrm{~mL})$ were diluted by adding $1 \mathrm{~mL}$ of mobile phase and filtered using a $0.45-\mu \mathrm{m}$ PTFE syringe filter before analyzing. Samples of AS were filtered with a $0.45-\mu \mathrm{m}$ PTFE syringe filter. Fresh dissolution medium $(3 \mathrm{~mL}$ ) was replaced into each vessel after sampling. All samples were analyzed using a validated HPLC method $(14,15)$. Adsorption of the drugs onto the syringe and filter was evaluated before the dissolution studies.

Residual amounts of BD and AS on the membrane holder were determined after dissolution testing by washing the membrane and the impaction insert with mobile phase $(5 \mathrm{~mL})$ before analyzing. The total amount of BD and AS initially loaded on the membrane holder was back-calculated using the sum of cumulative release amounts of API plus the remaining quantity of API on the membrane holder. The percent drug release of $A S$ and $B D$ was calculated by dividing the amount of API released by the drug mass initially loaded on the membrane holder.

\section{Validated HPLC Analysis of BD and AS in the Dissolution Media}

Samples were analyzed using a Waters HPLC system (Waters Co., Milford, MA) with UV detection. The system consisted of a 717plus autosampler, 2487 dual-wavelength detector, 1525 binary pump, and 1500 column heater.
Chromatography was performed using a Capcell Pak C18 column, $5-\mu \mathrm{m}$ particle size, $4.6 \times 250 \mathrm{~mm}$ (Shiseido Fine Chemicals, Tokyo, Japan) for BD and an Alltima C18 column, 5- $\mu \mathrm{m}$ particle size, $4.6 \times 150 \mathrm{~mm}$ (Grace, Deerfield, IL) for AS. The mobile phase for BD was methanol/ acetonitrile/water/acetic acid (50:10:28.3:1.7) at a flow rate of $1.7 \mathrm{~mL} / \mathrm{min}$, and the UV detection wavelength was $242 \mathrm{~nm}$ (14). The mobile phase for AS was methanol/0.1\% ammonium acetate acetonitrile $(\mathrm{pH} 4.5)(45: 55)$ at a flow rate of $0.8 \mathrm{~mL} / \mathrm{min}$, and the UV detection wavelength was $276 \mathrm{~nm}$ (15). The column temperature for both APIs was $25^{\circ} \mathrm{C}$, and the sample injection volumes were $100 \mu \mathrm{L}$ for $\mathrm{BD}$ samples and $20 \mu \mathrm{L}$ for AS samples.

\section{Statistical Analysis}

Data were expressed as the mean $\pm \mathrm{SD}$. The statistical differences of release rates were studied by calculating a similarity factor, $f_{2}(16)$. For curves to be considered similar, $f_{2}$ values should be close to 100 . Generally, $f_{2}$ values greater than 50 (i.e., 50-100) ensure sameness or equivalence of the two curves (16).

\section{RESULTS AND DISCUSSION Dissolution Apparatus}

The membrane holder was placed at the bottom of each vessel, release-surface side up, with the distance between the bottom edge of the paddle and the surface of the membrane holder maintained at $20 \pm 2 \mathrm{~mm}$ (Figure 1A).The distance between the paddle and the surface of the membrane holder can be adjusted within a range that allows the paddle to effectively remove released drug from the exposed membrane surface and provides continuous circulation to the media in the vessel.

The mechanism of this dissolution method can be explained by a dissolution-diffusion-controlled drug release from the membrane holder. During the dissolution process, the dispersed drug within the membrane holder undergoes dissolution as dissolution medium migrates through the pores on the membrane surface, and the dissolved drug then releases out to the reservoir by diffusion (13). Therefore, membrane properties such as pore size, pore tortuosity, and membrane thickness have a profound effect on the release behavior of each drug. A PC membrane was selected as the diffusion barrier (13). The PC membrane surface constitutes a perfect sink for the released drug when used in this fashion. Dissolution media is able to diffuse rapidly into the new membrane holder and reach the drug inside through the numerous pores, subsequently allowing dissolved drug to be immediately removed from the exposed membrane surface under suitable hydrodynamic conditions. In the previous study reported by this group (13), the PC membrane demonstrated an almost twofold increase in drug release rate when compared with cellulose acetate (CA) dialysis membranes. This disparity is attributed to the differences in the physical structure of the two membranes. PC membranes are thin (approximately $6 \mu \mathrm{m}$ ) 
but still relatively robust for their handling requirements. Additionally, they do not swell, do not create air bubbles, have a well-defined uniform pore size $(17,18)$, and consist of homogeneous $0.05-\mu \mathrm{m}$ non-tortuous cylindrical pores on the surface that allow free diffusion of dissolved drug and dissolution medium.

\section{Dissolution Procedure \\ Dose Collection}

Aerodynamic particle classification was achieved with the NGI for both Ventolin HFA and Pulmicort Flexhaler to select a suitable particle size cutoff and amount of drug loading for the following dissolution study. Unlike usual dissolution methods, drug loading into the membrane holder is achieved by particle impingement activity; thus, the particle deposition profile on each dose-collection plate is essential in determining the amount of initial drug loading and the particle size cutoff. Collection plate 4 for Pulmicort Flexhaler and plate 5 for Ventolin HFA were selected for the dissolution method validation study, because these consistently displayed the maximum deposition for each product, as shown in Figure 3. Additionally, the median aerodynamic particle size values $\left(D_{50}\right)$ for collection plates 4 and 5 of the NGI were 1.66 and $1.36 \mu \mathrm{m}$ at flow rates of $60 \mathrm{~L} / \mathrm{min}$ and $30 \mathrm{~L} / \mathrm{min}$, respectively, which fall into the respirable particle size range (1-5 $\mu \mathrm{m})(19)$.

To enable the dissolution studies, the impaction insert of the new membrane holder was inserted into the preselected NGI dissolution cup of the NGI (impaction plates before and after device actuation impingements are shown in Figure 2). The amounts of BD or AS collected on the impaction insert of the membrane holder are presented in Table 1. The membrane holder assembly used in this study was designed to facilitate maximum particle collection over a uniform collection area $\left(18 \mathrm{~cm}^{2}\right)$.

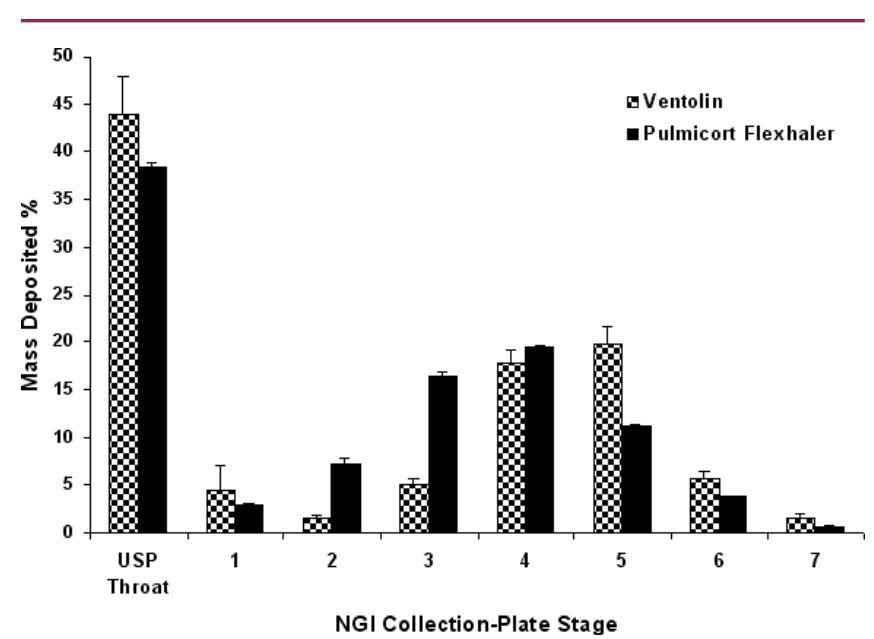

Figure 3. Particle deposition at dose plate for Ventolin HFA and Pulmicort Flexhaler. Ventolin HFA and Pulmicort Flexhaler were actuated 5 times at $30 \mathrm{~L} / \mathrm{min}$ and $60 \mathrm{~L} / \mathrm{min}$, respectively. The error bars indicate the standard deviation of three tests.
Table 1. Amount of Drug Loaded on Dose-Collection Plate 4 (BD) and Plate 5 (AS) for Dissolution Studies

\begin{tabular}{lcccc}
\hline \multicolumn{2}{c}{ Pulmicort Flexhaler (BD) } & & \multicolumn{2}{c}{ Ventolin HFA (AS) } \\
\hline $\begin{array}{l}\text { Number of } \\
\text { Actuation (T) }\end{array}$ & $\begin{array}{c}\text { Amount of } \\
\text { Loading }(\boldsymbol{\mu g})\end{array}$ & & $\begin{array}{c}\text { Number of } \\
\text { Actuation }(\mathrm{T})\end{array}$ & $\begin{array}{c}\text { Amount of } \\
\text { Loading }(\boldsymbol{\mu g})\end{array}$ \\
\hline 1 & $29.4 \pm 5.1$ & 15 & $259.5 \pm 42.4$ \\
\hline 2 & $55.2 \pm 3.8$ & 20 & $320.7 \pm 17.7$ \\
\hline 5 & $151 \pm 12.7$ & & \\
\hline 10 & $224 \pm 5.3$ & & \\
\hline
\end{tabular}

The amount of loading was calculated by adding the quantity of API remaining on the membrane holder to the total quantity of API released from the holder.

Particles having different particle size distributions can be easily collected by replacing a standard collection plate with the NGI dissolution cup equipped with the impaction insert of the new membrane holder.

Additionally, this method can be used to examine the dissolution behaviors of inhalation dosage forms with similar aerodynamic particle distributions by selecting drug particles accumulated in the same collection plate. The dissolution of particles having a similar aerodynamic size distribution should provide more interformulation discrimination.

Dissolution profiles of BD particles having different particle size cutoffs were conducted to evaluate particle size dependency in the dissolution process. For the dissolution study, an average $25 \mu \mathrm{g}$ of BD powder was collected on the impaction insert placed in collection plates $2-5$. Figure 4 shows that the release profiles of

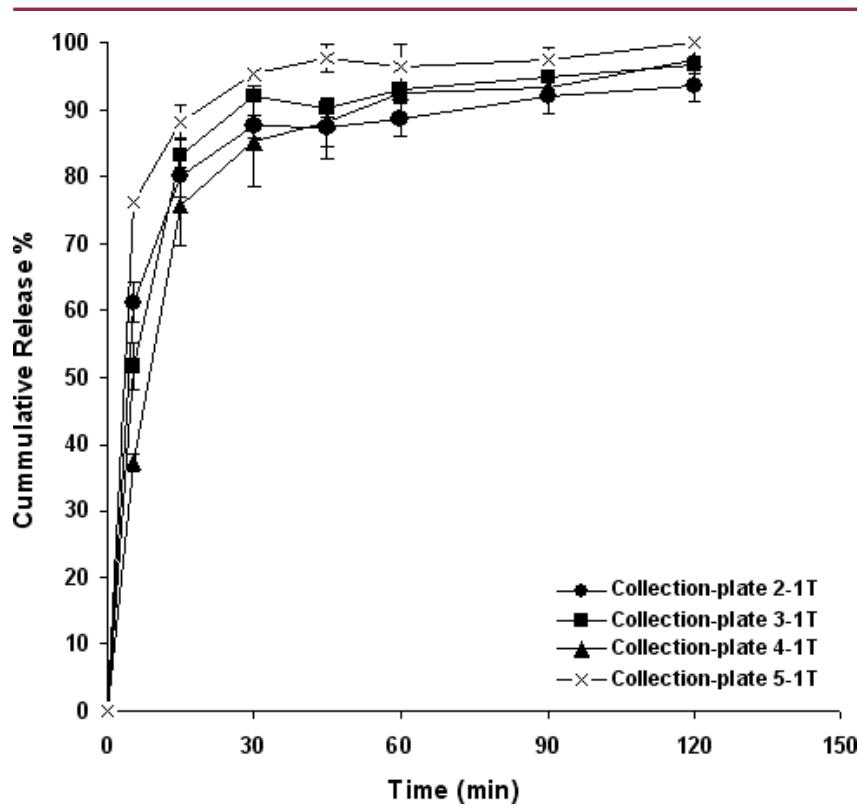

Figure 4. Release profiles of BD for dose-collection plates 2-5. T is the number of actuations. The error bars indicate the standard deviation of three tests. 
Table 2. Similarity Factors $\left(f_{2}\right)$ Between Two Dissolution Curves of BD Collected from Pulmicort Flexhaler Device

\begin{tabular}{|c|c|c|c|c|c|c|c|c|}
\hline$R_{t}$ & $T_{t}$ & $f_{2}$ & $R_{t}$ & $T_{t}$ & $f_{2}$ & $R_{t}$ & $T_{t}$ & $f_{2}$ \\
\hline \multirow{2}{*}{ SLF -1T } & PBS- 1T & 63.4 & \multirow{3}{*}{ SLF-1T } & mPBS-5T (0.02\%) & 25.1 & \multirow{3}{*}{ Stage 4 (SLF-1T) } & Stage 2 (SLF-1T) & 52.5 \\
\hline & Phosphate Buffer-1T & 51.6 & & tPBS-5T (0.02\%) & 40.7 & & Stage 3 (SLF-1T) & 59.7 \\
\hline & & & & tPBS-5T (0.2\%) & 64.7 & & Stage 5 (SLF-1T) & 40.6 \\
\hline
\end{tabular}

$R_{t}:$ reference performance

$T_{t}:$ test performance

$T$ : number of actuations

particles accumulated on $\mathrm{NGI}$ collection plates 2, 3, and 4 reached $80 \%$ after $15 \mathrm{~min}$, with a significant difference in initial release rates. The similarity factors $\left(f_{2}\right)$ are 52.5 for collection plate 2 and 59.7 for collection plate 3 when individually compared with the dissolution profile of the reference test, collection plate 4 (Table 2). With particles accumulated on collection plate 5 , the overall dissolution profile is not equivalent to the reference test (collection plate 4$) ; f_{2}$ is 40.6 . That is because those smaller particles have better wettability and dissolve quickly due to their larger surface area, as explained in the previous study (13). Dose-collection plate cutoff particle sizes calibrated from the $\mathrm{NGI}$ are $4.46,2.82,1.66,0.94,0.55$, and $0.34 \mu \mathrm{m}$ at an inlet flow rate of $60 \mathrm{~L} / \mathrm{min}$ for collection plates $2-7$, respectively (19).

\section{Hydrodynamic Conditions}

Dissolution tests of BD were conducted at paddle speeds of 50,75 , and $100 \mathrm{rpm}$. Dissolution profiles obtained at a rate of $75 \mathrm{rpm}$ were the most consistent, as shown in Figure 5. For the paddle apparatus, $50 \mathrm{rpm}$ is

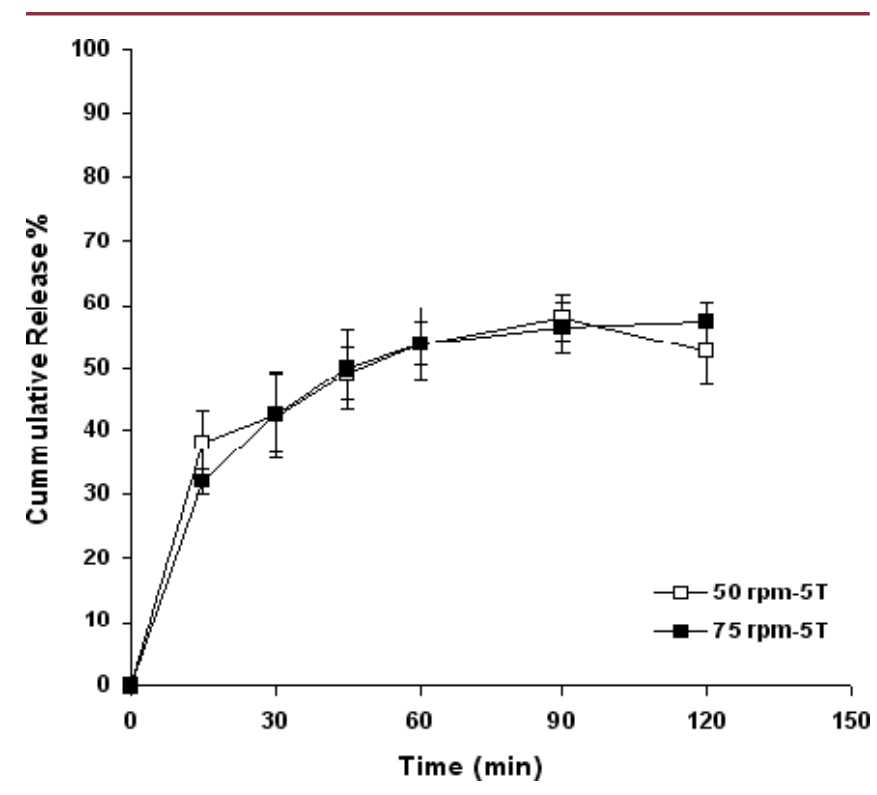

Figure 5. Release profiles of BD at rotating speeds of $50 \mathrm{rpm}$ and $75 \mathrm{rpm}$. T is the number of actuations. The error bars indicate the standard deviation of three tests. most commonly used; however, a higher paddle speed was required because of the "dead" volume between the membrane holder and the bottom of the vessel. The dissolution medium circulates through the gap between the holder and the vessel wall around the holder. However, because of the narrow gap, the circulation of medium was somewhat hindered, and a higher agitating speed was required to provide better circulation of the medium under the holder. The release profile of BD at an agitating speed of $100 \mathrm{rpm}$ was not plotted because an eddy was observed.

\section{Dissolution Media}

The dissolution profiles of BD (Pulmicort Flexhaler) in simulated lung fluid (SLF), 0.2 M phosphate buffer, and phosphate buffered saline (PBS) were tested. The dissolution profiles from the three media were similar, as shown in Figure 6A. Table 2 shows the $f_{2}$ values between PBS and phosphate buffer as 63.4 and 51.6, respectively, when compared with SLF. Therefore, either phosphate buffer or PBS could be used as an alternative dissolution medium. The SLF was first developed by Moss (20) and was described as being similar to actual lung fluid in ionic composition and $\mathrm{pH}$. SLF has been used in previously published in vitro dissolution studies because of its similarity in composition to actual lung fluid $(6,9,20)$. However, SLF media may not be preferable for routine QC studies for inhalation products because of its low buffering effect. In particular, the use of SLF media is not recommended for evaluating inhalation dosage forms that show $\mathrm{pH}$ dependency or sustained-release dissolution profiles. As shown in Figure $6 \mathrm{~B}$, the $\mathrm{pH}$ of SLF increases from 7.4 to 8.8 in $24 \mathrm{~h}$ without continuous $\mathrm{CO}_{2}$ bubbling. It has been reported that the $\mathrm{pH}$ of SLF media significantly increased without $\mathrm{CO}_{2}$ bubbling (20).

A medium volume of $300 \mathrm{~mL}$ was used for dissolution studies. This was determined to be a minimum volume needed to occupy the $20 \pm 2 \mathrm{~mm}$ distance between the membrane holder surface and the bottom edge of the paddle, as shown in Figure 1A. The medium volume may be raised to $900 \mathrm{~mL}$, depending on the concentration and sink condition of the drug. The dissolution of drug from the membrane holder occurs by diffusion of dissolution medium through the pores on the membrane surface. The amount of dissolution medium that diffused through the 

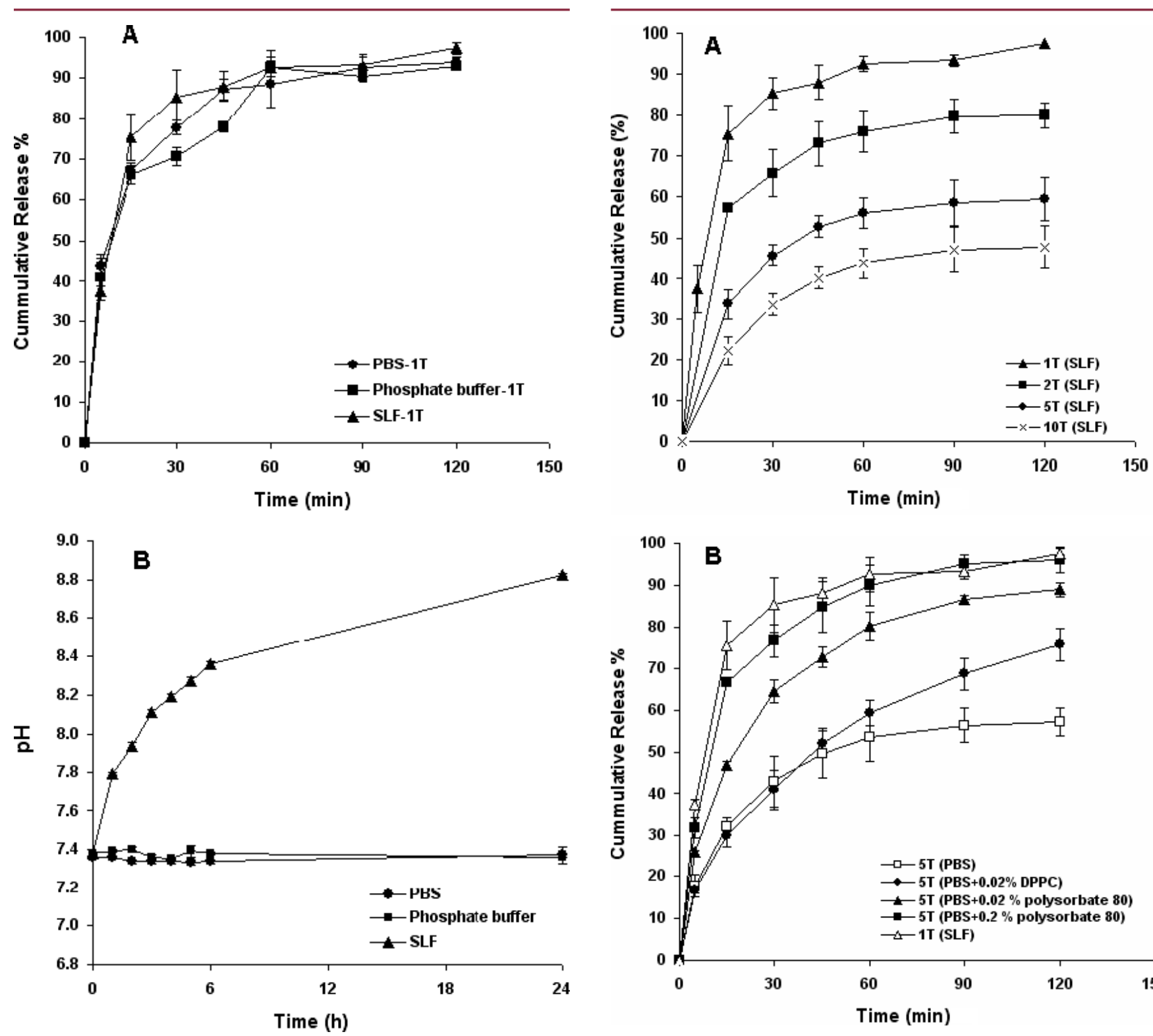

Figure 6. (A) Release profiles of BD in three different dissolution media, $P B S$, $0.2 \mathrm{M}$ phosphate buffer, and SLF. (B) pH profiles of $P B S, 0.2 \mathrm{M}$ phosphate buffer, and SLF media without continuous $\mathrm{CO}_{2}$ bubbling for $24 \mathrm{~h}$. T is the number of actuations. The error bars indicate the standard deviation of three tests.

pores was mainly determined by the paddle speed and pore size on the membrane surface and not by the amount of medium in the vessel. Normally, for the paddle and basket apparatus, the range of medium volume is 500-1000 mL, where $900 \mathrm{~mL}$ is most common volume. However, the dissolution system may be optimally less than sink conditions for test compounds to approximate the dissolution behavior of formulations in the lung, since the volume of lung fluid is extremely small, approximately $10-20 \mathrm{~mL} / 100 \mathrm{~m}^{2}$ of surface $(1,2)$. The membrane holder used in this study was designed to maintain a very thin liquid layer between the membrane and test powders.

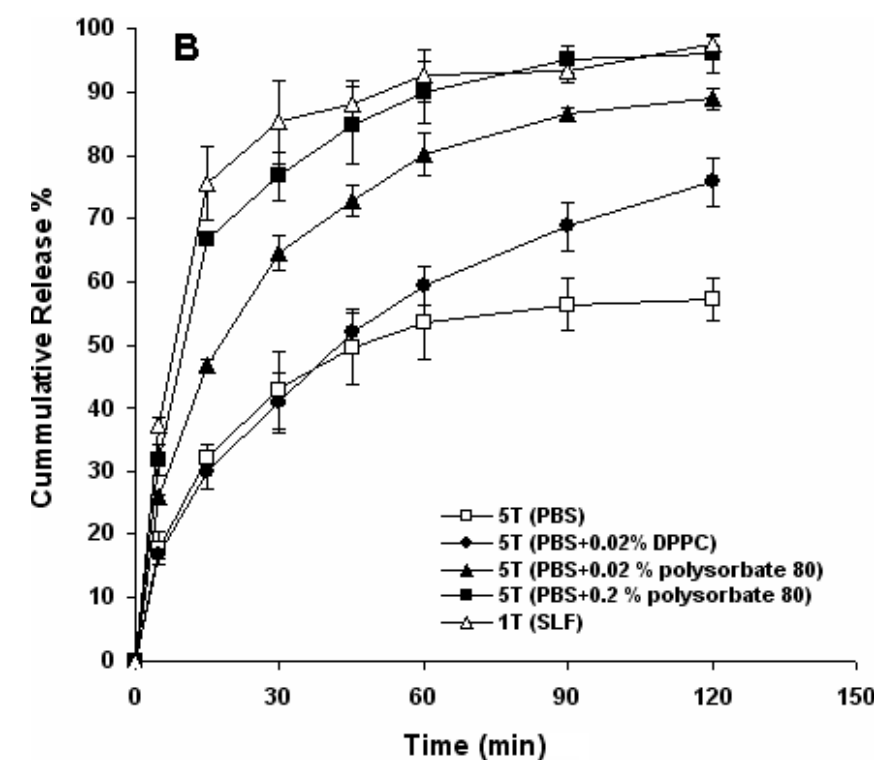

Figure 7. (A) Release profiles of $B D$ from the holder having different drug loading in SLF media for dose plate 4. (B) Release profiles of BD in PBS media containing $0.02 \%$ DPPC, $0.02 \%$ polysorbate 80 , and $0.2 \%$ polysorbate $80 . T$ is the number of actuations. The error bars indicate the standard deviation of six tests.

Dissolution medium was continuously exchanged from the vessel reservoir to the drug particles enclosed inside the holder by agitation, and a very thin liquid layer was maintained to dissolve drug particles (13). In other words, a dynamic equilibrium was rapidly established for solute exchange, following the commencement of a given dissolution test with the membrane holder.

\section{Amount of Drug Loading}

The amount of drug loading on the membrane holder showed significant influence on the drug release rate, especially for the Pulmicort Flexhaler, which contains the 


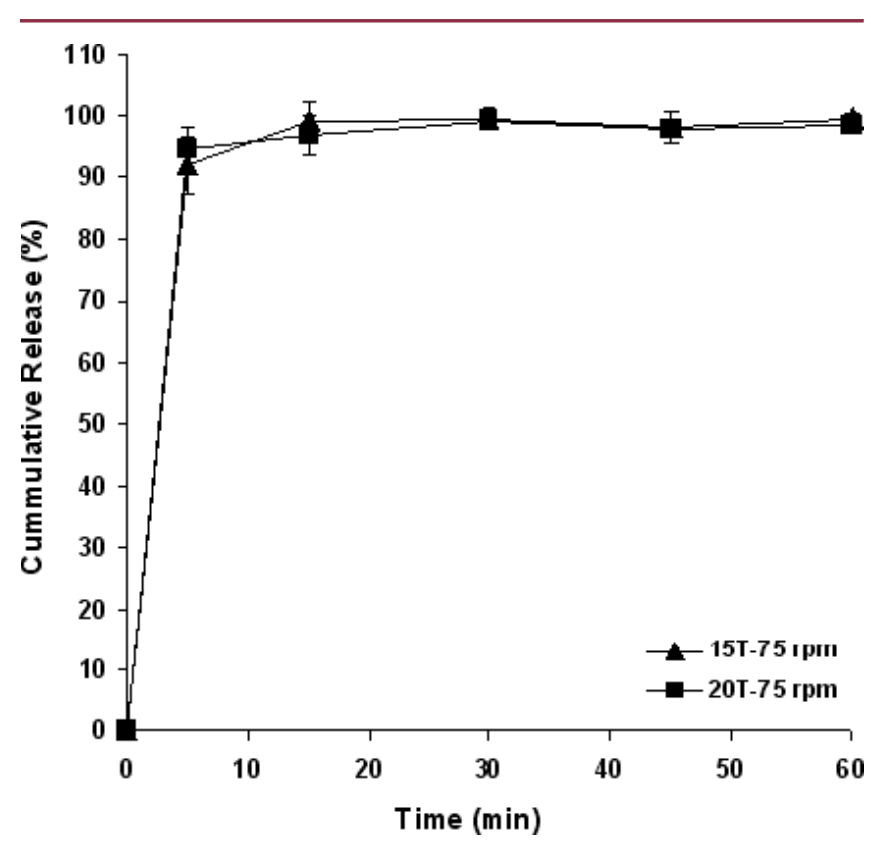

Figure 8. Release profiles of AS in SLF media. T is the number of actuations. The error bars indicate the standard deviation of six tests.

hydrophobic steroid BD. The amount of drug loaded on the membrane holder is summarized in Table 1. Figure 7A shows that $80 \%$ dissolution was achieved in 30 min when $29.4 \mu \mathrm{g}(1 \mathrm{~T})$ of drug is loaded. As the loading amount increased from $29.4 \mu \mathrm{g}(1 \mathrm{~T})$ to $224 \mu \mathrm{g}$ (10T), the dissolution rate significantly slowed. In contrast to $B D$, the dissolution rates of Ventolin HFA, which contains hydrophilic AS, did not display the same degree of dose dependency, because $90 \%$ dissolution was achieved in $5 \mathrm{~min}$ for all the test samples, as shown in Figure 8. This indicates that the loading amount may determine powder wetting inside the holder, and the significance of this was dependent on the hydrophobicity of the API used. When the device is actuated, dispersed powders form a powder bed on each dose-collection plate, and the thickness of the powder bed is dependent on the amount of drug loaded on each dose-collection plate. A larger amount of drug loaded on the plate corresponds to a thicker powder bed. Given a thicker load of powder bed on the membrane holder, there will be more dissolution-diffusion activity required to release all of the drug from the inner space of the membrane holder because the dissolution of loaded drug gradually progresses inward layer by layer until all solid drug particles have been wetted and dissolved. Consequently, the release rate of molecules is governed by the thickness of the drug layer and by the solubilization rate of that drug layer. Ideally, the device needs to be actuated one time to obtain well-dispersed particles in an approximately single layer. However, the thickness of the powder bed can also be varied according to the drug dose. For instance, a one-time actuation of a high-dose formulation can create a thicker powder bed than a one-time actuation of a low-dose formulation.

Additionally, in some cases, multiple actuations may be necessary to obtain quantifiable drug concentration in the medium since at least $300 \mathrm{~mL}$ of dissolution medium is required to conduct the dissolution study. Care must be taken when the loading dose is high or is obtained by multiple device actuations, especially for poorly water-soluble drugs.

For testing very poorly soluble compounds, dissolution media may contain a percentage of surfactant to enhance drug solubility (16). To improve the wettability of $\mathrm{BD}$ particles and to help their solubilization, two different surfactants, polysorbate 80 and dipalmitoyl phosphatidylcholine (DPPC), were added to PBS media. Polysorbate 80 provided a better solubilization effect to the BD powder than DPPC, as shown in Figure 7B.DPPC may help powder wetting; however, the effect was not significant. The initial drug release rate of BD in $\mathrm{mPBS}$ during the first 45 min was similar to that in SLF media.

DPPC is a main component of lung surfactant, and the effect of lowering the surface tension at the air-water interface is essentially performed by a DPPC monolayer (21). Because of their similarity in composition to actual lung fluid, dissolution media containing $0.02 \%$ of DPPC have been widely used to predict the solubility and solubilization process of inhalation formulations that have very low aqueous solubility. However, in this study using the new membrane holder, DPPC in the media may not be able to freely diffuse and reach the drug particles inside the membrane holder because DPPC forms liposomal aggregates in aqueous media. These liposomal aggregates have a bigger particle size distribution than the membrane pore size on the surface. Thus, although wettability of BD powders was somewhat increased by adding DPPC, significant wetting or solubilizing of BD was not found over the test period, as shown in Figure $7 \mathrm{~B}$ and Table 2. PBS containing $0.2 \%(\mathrm{w} / \mathrm{v})$ polysorbate 80 was an ideal dissolution medium for evaluating $\mathrm{BD}$ actuated five times, as $f_{2}$ was 64.7 when compared with the dissolution curve of BD actuated 1 time, which is assumed to be an ideal dose for dissolution. The concentration of surfactant must be justified by showing profiles at several different concentrations for a variety of different dose loadings, and dissolution profiles must be compared with that of a standard sample to determine a suitable concentration of surfactant.

\section{CONCLUSION}

A new, easy-to-use dissolution membrane holder for evaluating the in vitro dissolution behavior of inhalation formulations was designed. The dissolution rates of commercially available drug formulations were successfully estimated by analyzing the amount of drug released from this attachment for the NGI. This dissolution method may be used for quality control studies for various dry-powder inhalers; in particular, the in vitro dissolution profiles of a drug may provide an estimate of its dispersion 
characteristics, which directly relate to the device or aerosol performance.

\section{REFERENCES}

1. Gray, V. A.; Hickey, A. J.; Balmer, P.; Davies, N. M.; Dunbar, C.; Foster, T. S.; Olsson, B. L.; Sakagami, M.; Shah, V.P.; Smurthwaite, M. J.; Veranth, J. M.; Zaidi, K. The Inhalation Ad Hoc Advisory Panel for the USP Performance Tests of Inhalation Dosage Forms. Pharm. Forum 2008, 34 (4), 1068-1074.

2. Patton, J.S. Mechanisms of macromolecule absorption by the lungs. Adv. Drug Deliver. Rev. 1996, 19 (1), 3-36.

3. Asada, M.;Takahashi, H.; Okamoto, H.; Tanino, H.; Danjo, $\mathrm{K}$. Theophylline particle design using chitosan by the spray drying. Int. J. Pharm. 2004, 270 (1-2), 167-174.

4. Jaspart, S.; Bertholet, P.; Piel, G.; Dogne, J. M.; Delattre, L.; Evrard, B. Solid lipid microparticles as a sustained release system for pulmonary drug delivery. Eur. J. Pharm. Biopharm. 2007, 65 (1), 47-56.

5. Learoyd, T. P.; Burrows, J. L.; French, E.; Seville, P.C. Chitosan-based spray-dried respirable powders for sustained delivery of terbutaline sulfate. Eur.J.Pharm. Biopharm. 2008, 68, (2), 224-234.

6. Davies, N. M.; Feddah, M. I. R. A novel method for assessing dissolution of aerosol inhaler products. Int. J. Pharm. 2003, 255 (1-2), 175-187.

7. Cook, R. O.; Pannu, R. K.; Kellaway, I.W. Novel sustained release microspheres for pulmonary drug delivery. J. Control. Release 2005, 104 (1), 79-90.

8. McConville, J.T.; Patel, N.; Ditchburn, N.; Tobyn, M. J.; Staniforth, J. N.; Woodcock, P. Use of a novel modified TSI for the evaluation of controlled-release aerosol formulations. I. Drug Dev. Ind. Pharm. 2000, 26 (11), 1191-1198.

9. Ansoborlo, E.; Guilmette, R. A.; Hoover, M. D.; Chazel, V.; Houpert, P.; Henge-Napoli, M. H. Application of in vitro dissolution tests to different uranium compounds and comparison with in vivo data. Radiat. Prot.Dosim. 1998, 79 (1-4), 33-37.

10. Sdraulig, S.; Franich, R.; Tinker, R. A.; Solomon, S.; O'Brien, R.; Johnston, P. N. In vitro dissolution studies of uranium bearing material in simulated lung fluid. J. Environ. Radioactiv. 2008, 99 (3), 527-538.
11. Kwon, M. J.; Bae, J.H.; Kim, J. J.; Na, K.; Lee, E. S. Long acting porous microparticle for pulmonary protein delivery. Int. J. Pharm. 2007, 333 (1-2), 5-9.

12. Ungaro, F.; De Rosa, G.; Miro, A.; Quaglia, F.; La Rotonda, M. I. Cyclodextrins in the production of large porous particles: Development of dry powders for the sustained release of insulin to the lungs. Eur. J. Pharm. Sci. 2006, 28 (5), 423-432.

13. Son, Y. J.; McConville, J.T. Development of a standardized dissolution test method for inhaled pharmaceutical formulations. Int. J. Pharm. 2009, 382 (1-2), 15-22.

14. Feddah, M. R.; Brown, K. F.; Gipps, E. M.; Davies, N.M. In-Vitro Characterisation of Metered Dose Inhaler Versus Dry Powder Inhaler Glucocorticoid Products: Influence of Inspiratory Flow Rates. J. Pharm. Pharm. Sci. 2000, 3 (3), 317-324.

15. Harris, J. A.; Stein, S. W.; Myrdal, P. B. Evaluation of the TSI aerosol impactor 3306/3321 system using a redesigned impactor stage with solution and suspension metered-dose inhalers. AAPS PharmSciTech 2006, $7(1), \mathrm{E} 1-\mathrm{E} 8$.

16. <1092> The Dissolution Procedure: Development and Validation. In United States Pharmacopeia and National Formulary USP 32-NF 27. The United States Pharmacopeial Convention, Inc.: Rockville, MD, 2009.

17. Marre, S.; Palmeri, J. Theoretical study of aerosol filtration by nucleopore filters: The intermediate crossover regime of Brownian diffusion and direct interception. J. Colloid Interf. Sci. 2001, 237 (2), 230-238.

18. Rzepka, S.; Neidhart, B. Transport processes through track-etch membrane filters in a reagent delivery cell. Fresen. J. Anal. Chem. 2000, 366 (4), 336-340.

19. Marple, V. A.; Roberts, D. L.; Romay, F. J.; Miller, N. C.; Truman, K. G.; Holroyd, M. J.; Mitchell, J. P.; Hochrainer, D. Next generation pharmaceutical impactor (A new impactor for pharmaceutical inhaler testing). Part I: Design. J. Aerosol Med. 2003, 16 (3), 283-299.

20. Moss, O. R. Simulants of lung interstitial fluid. Health Phys. 1979, 36, 447-448.

21. Veldhuizen, R.; Nag, K.; Orgeig, S.; Possmayer, F. The role of lipids in pulmonary surfactant. BBA-Mol. Basis Dis. 1998, 1408 (2-3), 90-108. 